\title{
Clostridium sporogenes
}

National Cancer Institute

\section{Source}

National Cancer Institute. Clostridium sporogenes. NCI Thesaurus. Code C86294.

A species of anaerobic, Gram positive, rod shaped bacteria assigned to the phylum

Firmicutes. This species is proteolytic, does not produce botulinum toxin, is beta-

hemolytic and positive for indole. C. sporogenes is pathogenic and culture is associated with a foul odor. 